\title{
LLORAR DELANTE DE UNA PANTALLA PLANA. EMOCIONES \\ COMPARTIDAS EN UN LOCUTORIO
}

\section{CRYING IN FRONT OF A FLAT SCREEN. SHARED EMOTIONS IN A TELEPHONE CALLING SHOP}

\author{
Simone Belli \\ Universidad Autónoma de Madrid. España/Spain \\ simone.belli@uam.es \\ Adriana Gil Juárez \\ Universidad Rovira i Vigili, Tarragona. España/Spain \\ adriana.gil@urv.cat
}

Recibido/Received: 02/02/2011

Aceptado/Accepted: 30/09/2011

\section{RESUMEN}

En este artículo presentaremos una parte del análisis de entrevistas realizadas a usuarios y usuarias de locutorios en Barcelona, Manchester y Milán. El énfasis se ha puesto en el análisis de las emociones ligadas al uso de estas tecnologías en estos espacios específicos. Nos centraremos en comprenderlos como espacios públicos en continuo movimiento y evolución, que generan emociones estrictamente relacionadas con ellos, y construidas a través del lenguaje. Utilizando el concepto de performance, se explora cómo el habla crea una necesidad de construir emociones particulares, que no existen antes de su performance.

\section{PALABRAS CLAVE}

Emociones, performance, TIC, locutorios, intimidad.

\section{SUMARIO}

1. Introducción. 2. ¿Qué es una performance emocional? 3. Encontrar la performance en el discurso. 4. Llorar delante de una pantalla plana. 5. Conclusiones. Bibliografia.

\begin{abstract}
In this paper we will present a piece of the analysis of interviews realized to users of Telephone calling shops in Barcelona, Manchester and Milan. The emphasis has been put in the analysis of the emotions tied to the use of ICT in these specific spaces. We will focus on understanding these spaces as public spaces in continuous movement and evolution, which generate emotions strictly related to them. Using the concept of performance, we explore how speech creates a need for the construction of particular emotions, which do not exist before they performance.
\end{abstract}

\section{KEYWORDS}

Emotions, performance, ICT, Telephone calling shops, intimacy.

\section{CONTENTS}

1. Introduction. 2. What is a emotional performance? 3. Meet performance in the discourse. 4. Crying in front of a flat screen. 5. Conclusions. References. 


\section{INTRODUCCIÓN}

Cada vez es más sencillo el acceso de los y las ciudadanos/as a las tecnologías de la información y de la comunicación, las TIC, gracias a la existencia de diferentes espacios públicos que permiten su uso. Estos lugares van destacando, al igual que las TIC, por su presencia habitual y en alza en nuestros escenarios urbanos. En el contexto de los locutorios, hay prácticas que son una elección continua y cotidiana, que tienen una función importante y esencial para la vida social y la relación con los demás: la utilización humana y social del espacio, que nosotros consideramos como un espacio construido a través de la performance emocional. Es por ello que en este trabajo nos interesaremos por las emociones compartidas en el uso de estos espacios.

En nuestras investigaciones sobre el uso de Tecnologías de la Información y Comunicación (TIC) por parte de usuarios de locutorios y "cibercafés" (Belli, Íñiguez, 2008; Belli, Martínez, Peñaranda, Vítores e Íñiguez, 2007; Feliu, 2006; Garay, Martínez, y Peñaranda, 2004; Gil-Juárez, 2006; Gil-Juárez, Feliu, 2004; Gil-Juárez, Feliu, en prensa; Martínez, Peñaranda, 2006), hemos entendido cómo estas tecnologías son fundamentales en la vida cotidiana de cada individuo.

El énfasis en este trabajo, se ha puesto en el análisis de las emociones relacionadas con el uso de estas tecnologías en estos espacios específicos. Utilizando el concepto de performance (Butler, 1993), se explora cómo el habla crea una necesidad de construir emociones particulares que no existían antes de su performance. Estos actos o "fabricaciones" se consideran naturales a través de su ejecución repetida en el tiempo, en un conjunto de múltiples interacciones sociales cotidianas.

Para poder entender esta performance en los discursos es necesario utilizar una "herramienta" ad hoc, en nuestro caso es el Membership Categorization Analysis tal y como lo entiende la escuela de Manchester (Leudar, 1995,1998). La particularidad de esta perspectiva del MCA es que reconoce a las emociones como una variación y cambio continuo en el lenguaje, una iteración constante y progresiva, es decir, una performance. Con esta particular simbiosis parece abrirse una nueva área de estudios entre las emociones y las TIC, entendiendo la emoción como algo simbólico y que encuentra en la Lingüística su mejor expresión.

Presentaremos primero una breve descripción de lo que entendemos por performance emocional, que nos ayudará a entender el uso que la comunidad hace de los locutorios. En la segunda parte nos centraremos en la metodología utilizada para encontrar esta performance en el discurso, el Membership Categorization Analysis, según la concepción de la escuela de Manchester.

Con la finalidad de poder observar cómo cambia el uso que hacen de las TIC y la relación con las emociones que experimentan, en esta parte del trabajo hemos analizado 17 entrevistas a usuarios y usuarias de locutorios de tres ciudades europeas diferentes (Barcelona, Manchester y Milán) donde la presencia de estos espacios es masiva y creciente, de nacionalidades distintas, y tanto de inmigrantes como no inmigrantes (Belli, Íñiguez, 2008; Belli, Martínez, Peñaranda, Vítores e Íñiguez, 2007).

Finalmente concluimos con la importancia de entender los locutorios como espacios públicos en continuo movimiento y evolución, que generan emociones estrictamente relacionadas con ellos, y construidas gracias al lenguaje. 


\section{2. ¿QUÉ ES UNA PERFORMANCE EMOCIONAL?}

Cuando se trata el tema de performance en los estudios postconstruccionistas el nombre de Judith Butler es probablemente el más importante. Butler $(1993,1997)$ trata el tema de las emociones como una evolución constante en los discursos, ofreciendo una mirada a las emociones con una óptica completamente innovadora a través el concepto de performance. Aunque en sus textos no aparece explícitamente el término "emoción", surge de manera espontánea en todos sus discursos y posturas.

Utilizando el concepto de performance (Butler, 2003) se explora cómo el habla crea una necesidad de construir emociones particulares: "Estos actos, gestos, promulgaciones, generalmente construidos, son performativos en el sentido que la esencia o identidad que se pretende expresar son fabricaciones constituidas y sostenidas a través los signos corporales y otros medios discursivos." (Butler, 2003:136). De esta manera la emoción es una performance producida a través de estas fabricaciones, actos internamente discontinuos. Es decir, las emociones no existen antes de su performance, y el éxito de la copia, la "repetición" de una emoción previamente constituida nunca puede ser invocada para reproducir fielmente a aquella.

Estos actos o "fabricaciones" se consideran naturales a través de la ejecución repetida en el tiempo, en un conjunto de múltiples interacciones sociales cotidianas. Estos actos performativos están abiertos a constante transformaciones y redefiniciones. Los actos o "fabricaciones" (Butler, 2003) eventualmente devienen normativas, y éstas pueden ser vistas como naturales.

Otro concepto muy importante para Butler es el de iteración (Butler, 1993). La autora utiliza la teoría de la iterabilidad de Derrida ya que le permite profundizar su concepto de performance:

"La performatividad no puede ser entendida fuera de un proceso de iterabilidad, una regulada y limitada repetición de normas. (...) Esta iterabilidad implica que la performance no es un acto, o evento, singular, sino que es una producción ritualizada (...)" (Butler, 1993:95). En definitiva, "Una repetición estilizada de actos" (Butler, 2003:140).

Nosotros exploramos en nuestra investigación cómo estas "fabricaciones" construyen emociones. Las emociones así concebidas son un fenómeno construido socialmente, una construcción identitaria donde quien habla performa la manera de comportarse (Butler, 2003). Como explicamos anteriormente, estas emociones no pre-existen antes de su performance y no pueden ser reutilizadas, categorizadas y citadas en un futuro (Gregson y Rose, 2000:438).

La performance emocional se puede entender pensando en una espiral que continuamente evoluciona e interacciona a través el lenguaje que producimos. Este lenguaje se articula y se constituye principalmente a través de la acción, es decir las actividades, y por consecuencia, en los verbos. Para describir esta nueva manera de acercarnos al tema de las emociones, necesitamos una herramienta "nueva", o por lo menos, una diferente manera de utilizar una herramienta ya existente. 


\section{ENCONTRAR LA PERFORMANCE EN EL DISCURSO}

La tarea fundamental para un investigador es encontrar la teoría en la práctica, es decir, en nuestro trabajo la performance en el discurso. Gracias al Membership Categorization Analysis, según la perspectiva de la escuela de Manchester, este objetivo lo hemos cumplido ya que hemos entendido cómo esta performance se va enfocando en la perspectiva del estudio de las emociones en las TIC. El Membership Categorization Analysis (MCA) es un análisis formal de los procedimientos empleados por la gente para dar sentido a otras personas y a sus actividades. Sacks (1992) formula el MCA a partir de los escritos de Chomsky y Lacan y su colaborador en aquellos años Emanuel Schegloff. Harold Garfinkel constituye la parte etnometodológica del MCA en la que se establece la representación de personas por categorías como la tarea principal del método. Las membership categories son constituidas por "categorías relacionadas a predicados", que pueden incluir actividades, disposiciones a actos, tareas, creencias y valores (Watson, 1987). Muchos de los trabajos en MCA utilizan categorías relativamente cristalinas (por ejemplo "madre", "hijo") y analizan cómo estas categorías son utilizadas en el habla. Pero uno de los problemas principales es que se tratan estas categorías como si fueran fijas. Este problema emerge, sobre todo, en las investigaciones de identidad social y políticas del cuerpo, ya que hay nuevas categorías que aparecen y desaparecen a lo largo del análisis. Esta particular caracterización pasa por el discurso y muchas veces lo involucra. Es decir, nos interesa ver cómo los participantes administran la relación entre categorías (Leudar, 1998). Sus administraciones están categorizadas en "colecciones", "clases" y "relaciones", pero lo que nos interesa principalmente para nuestra investigación es lo que plantea Leudar con el concepto de "distributed discursive network", es decir, cómo las categorías y las relaciones emergen interactivamente en el habla (Leudar, 1995, 1998; Leudar y Nekvapil, 1998).

La visión de Ivan Leudar se diferencia principalmente de la concepción de Sacks por su particular manera de entender las "categorías" de códigos. Según Leudar, estas "categorías" de códigos están en constante evolución en el interior de un discurso. Nunca son fijas, sino que cambian continuamente, y un miembro de un código muchas veces puede cambiar de categoría. Es un proceso dialógico, abierto y circular. La percepción de este cambio de categorías se debe a factores lingüísticos y lexicales producidos a través las prácticas sociales.

Otro aspecto importante en el planteamiento de Leudar es que las categorías tienen que ser activas, su concepción del MCA se define principalmente a partir este elemento básico. Imagina las categorías como un círculo, un sistema que se define, evoluciona constantemente, un sistema que siempre pasa de abierto a cerrado y de cerrado a abierto.

Un trabajo muy recomendable para comprender como se utiliza el Membership Category Analysis según esta perspectiva, nos lo ofrece el estudio sobre refugiados políticos de Leudar (1998). Leudar considera las categorías que cambian en los relatos de refugiados como categorías calificativas, en cuanto califican los miembros de cada categoría. Por ejemplo la categoría "madre", en un relato, puede pasar a ser categoría "hija". Este cambio es muy rápido, tanto que dura una expresión. El significado de estas categorías está directamente relacionado a las circunstancias en que estas categorías se encuentran. Estas categorías están directamente dependientes de las circunstancias, como por ejemplo las categorías "madre", "hija" De esta manera se considera y se utiliza cada categoría por cada persona. Como por ejemplo la categoría "checos" y "alemanes" durante la segunda guerra mundial, se ha notado 
que se puede pasar de la categoría "checo" a la categoría "alemán" en la misma entrevista en los estudios sobre refugiados.

Así que se puede considerar el habla como una actividad, ya que se la considera de una manera activa, y las mismas situaciones son activas, "talking in activity".

Justo porque la performance dibuja una espiral, hemos advertido la dificultad de utilizar herramientas clásicas a la hora de analizar nuestras entrevistas. No es posible hacer una fotografía de esta performance, y estudiarla como se estudian las ratas en un laboratorio, es decir enjauladas. Tenemos que dejar que evolucione esta performance, ver su trayectoria, verla en el habla en actividad.

La perspectiva de Manchester se centra en el estudio de los verbos, de las actividades, de las acciones, y haciendo un análisis de este tipo, nos encontramos con una especie de espiral. Unas espirales continuas que han substituido las líneas rectas y definidas de las relaciones entre sujeto y predicado. Una espiral que se caracteriza y se constituye por los verbos que surgen a lo largo del análisis, elementos que contienen nuestra performance emocional.

La performance emocional se dibuja a través de estas actividades y se sostiene por una serie de características, que a lo largo del análisis nos ayudan a definir la performance emocional que estamos manejando. Sólo de esta manera es posible ver esta performance, teniendo mucho cuidado de no utilizar herramientas nuevas con prácticas viejas. Hay que acercarse a esta metodología de una manera diferente para solucionar problemas nuevos.

Así que en el próximo apartado vamos a describir una trayectoria de esta performance, una trayectoria bien definida, que nos permite entender lo que hemos mencionado en esta primera parte.

\section{LLORAR DELANTE DE UNA PANTALLA PLANA}

A lo largo del siguiente análisis, nos hemos fijado en cómo aparecen performances emocionales es estos espacios públicos de los locutorios, sobre todo debido al uso que se hace de las Tecnologías de la Información y de la Comunicación en estos espacios. Un uso que constituye la actividad principal (Category bound activity) en nuestro análisis, hecha a través del Membership Categorization Analysis. Este uso que los entrevistados hacen de estas TIC, nos sirve para entender la performance emocional que nosotros definimos como la membership category de relacionarse con el Otro. Este Otro, dependiendo de los casos, es un familiar, un amigo o un desconocido, pero siempre se encuentra en el otro lado de nuestra pantalla, de nuestra TIC, estas pantallas que nos ven reír o llorar a según de lo que este Otro nos comunica. Estas actividades son la parte constituyente del análisis según esta perspectiva.

Consideramos esta performance estrictamente relacionada con el uso que hacemos de estas TIC, un uso social, y un uso estrictamente ligado al discurso, y producido a través de él. Un uso que nos permite introducir los elementos corporales en el lenguaje, según el planteamiento de Butler, transmitiendo por ejemplo un abrazo o un beso a través de un teclado.

Con la aparición de estas TIC en la sociedad contemporánea, en principio se pensó que eran maquinas frías, incapaces de transmitir emociones, por utilizar una expresión de Illouz (2007), emociones "congeladas", en el sentido que transmitían muy poco. Pero a lo largo de estos años, y sobre todo gracias a nuestro trabajo de analizar entrevistas de usuarios cotidianos de estas TIC, se ha entendido que estas maquinas producen verdaderas emociones, 
de ninguna manera frías (Belli, Gil, 2009). Esta performance ha pasado de considerarse una emoción fría, unemotional, a ser una emoción completamente verdadera, en cuanto la gente llora o ríe delante de una pantalla (Belli, S.; Íñiguez, L. 2008).

El uso de un medio frío, el ordenador, para poder comunicar con otras personas, la luz fría de la pantalla, para citar a Baudrillard (1990:153), un medio frío que emplaza a través de una seducción fría, el encanto narcisista de las nuevas tecnologías, el encanto frío de Internet. Pero esta no-emoción, unemotional, en el momento en que se relaciona con el uso que se hace de las TIC, con el comunicar con los demás, cambia, evoluciona, cumple una performance. Se transforma en una emoción real y presente. Esta evolución es debida al uso que se hace de estas tecnologías en estos espacios, un uso estrictamente discursivo y social: Un uso que nos sirve para comunicar con los demás, para relacionarnos con el Otro como podemos entender en los siguientes extractos que vamos a presentar.

En ellos, el uso que hacemos de las TIC para relacionarnos con los demás, se comprende plenamente. Este uso nos permite identificar como membership category principal la relación con el Otro. Esta relación se constituye a lo largo de la entrevista por tres características que son: la distinción de estar online y offline, la intimidad en estos dos espacios, y el concepto de identidades emocionales.

\subsection{Online y offline: mundos paralelos en el locutorio}

El uso que hacemos de estas TIC, es una extensión del propio cuerpo, bajo el concepto de cyborg (Haraway, 1990), pero siempre construido a través el lenguaje, en cuanto este uso siempre es discursivo. Gracias al lenguaje es posible definir esta extensión, este uso de las TIC, esta actividad. Se crea bajo esta performance del discurso, una iteración continúa por parte de los usuarios de estas tecnologías, para relacionarse con los demás, se va delineando, cumpliendo una iteración de asumir una condición de unemotional, fría, hasta ser emoción real, humana y con "color".

\section{Extracto 1}

323. -sí...mucho... porque quedan a tal hora....aquí horas en la tarde allá...mañana...con esto

324. guapísimo de la cámara...ves a tu mujer a tus hijos...o a tu familia...a tu madre o a tus

325. abuelos...y dos tres horas...ahí...llorando...algunos los verás llorar...decir cosas

326. muy...muy dulces...entonces tú escuchas y...te impacta... ¿no?

327. -¿CÓMO AFECTA ESO EN EL AMBIENTE? AY PERDONA...DI, DI...

328. \#\&-no yo una vez...me puse a emborracharme mirando la webcam con unos amigos que 329. estaba...y ah...salud... ((risas)) y bebían también ((risas)) y nos emborrachamos ((risas)) bueno

330. ya "chao" ((risas)) ....mata la añoranza...los...la añoranza...

En la línea 324 aparece el termino "cámara, y también "webcam" (328), términos tecnológicos que remiten al avance que estas TIC están teniendo en el escenario actual. Pero con la utilización de estas maquinas, vemos cómo éstas nos permiten acercarnos a nuestro contexto familiar, a nuestra gente lejana, a miles de kilómetros. La performance está en el percibir estas tecnologías cómo algo frío, pero que interacciona en el momento en que las utilizamos para comunicarnos. Se pasa de las emociones congeladas (Illouz, 2007) a emociones reales, como llorar delante de una pantalla (325), y decir cosas muy dulces a 
través del medio tecnológico (326). Estas tecnologías permiten compartir emociones entre usuarios, con extremos como "matar la añoranza" (330).

Sobre todo estas TIC nos permiten vivir nuestras vidas de manera online y offline, y muchas veces estos dos aspectos se juntan. El quedar con una persona en el mundo online implica que también en el mundo offline tengamos que planificarlo, por eso hay que quedar en un determinado espacio de tiempo: "porque quedan a tal hora...aquí horas en la tarde allá...mañana" (323). Por esta razón, nos ayuda a pensar en estos términos el concepto de mutante (Baricco, 2008). La figura del mutante nos sirve para explicar esta performance emocional de los usuarios de estas TIC en estos espacios específicos. En cuanto el mutante es un cyborg que ya ha olvidado las maquinas, ya no las ve como algo ajeno, y es capaz de extender el propio cuerpo discursivamente para comunicar con su propia gente. Estas maquinas le permiten comunicar una gama de emociones, llorar o reír, sin estas TIC no hubiera sido posible hacerlo a miles de kilómetros de distancia. Hemos dicho que estas maquinas nos permiten extender el propio cuerpo, sobre todo más allá del espacio real y físico, en cuanto discursivamente podemos ocupar espacio virtual para recrear nuestras propias emociones. Pensamos por ejemplo en cómo podemos enviar un abrazo o un beso a las personas que son importantes en nuestras vidas, pero que viven muy lejos de nuestro entorno. Esta acción la podemos hacer sólo a través el lenguaje, y sólo gracias a la performance emocional.

Para la entrevistada, utilizar esta webcam es una práctica cotidiana que le permite tener relaciones con su propia familia. Y cómo le pasa a ella, les pasa a todos los demás usuarios del locutorio (324-326). Estas prácticas cotidianas son las que nos permiten pensar a estos individuos que ya no utilizan estas tecnologías como cyborg, como maquinas ajenas y frías, a estos individuos que ya son mutantes, estas maquinas ya son parte de sus propias prácticas cotidianas. Practicas que le permiten relacionarse con el Otro, y la propia webcam desaparece, dejando sólo la comunicación emocional entre los dos lados de la pantalla, una continua performance emocional. Esta asimilación de las maquinas es posible sólo gracias al lenguaje, a través de eso podemos olvidarnos que estamos hablando a unas maquinas, y nos relacionamos directamente con la otra persona. Nos emborrachamos (329), matamos la añoranza (330), lloramos (325), son performance emocionales que continuamente varían según el contexto en que nos encontramos. Es imposible definirlas a priori en cuanto se producen en el contexto que el individuo construye hablando. Un continuo pasaje entre el mundo offline y el mundo online, donde muchas veces estas barreras se borran y es difícil definir donde aparecen y desaparecen determinadas emociones. Esta es la primera característica que emerge en esta membership category.

\subsection{Intimidades compartidas}

\section{Extracto 2}

333. -QUE CÓMO AFECTAN ESAS SITUACIONES EN EL AMBIENTE DEL

334. LOCUTORIO, ¿NO? EL HECHO QUE ALGUIEN PUEDA ESTAR

335. COMPARTIENDO..TENIENDO UNA CONVERSACIÓN...

336. .-te vuelves sensible...sales pero con la...con los ánimos muy bajos...a mi me

337. pasa...pero... porque ves tanta sensibilidad...tanta...a veces uno no aguanta...

338. (incomprensible) sales

339. \#- sí yo siempre que salgo de hablar con mi mamá...uy...es...

340. ./-claro porque es que ves tanta gente que...porque a veces estás en el otro teléfono y 
341. escuchas...porque habla muy fuerte y dices...y escuchas...porque son gente muy

342. humilde... "sí...yo te mando todo...me quedo yo con lo mínimo..." entonces...eso es lo

343. que...yo pienso que...cuando escucho algo así yo salgo más sensible...yo soy muy

343. sensible pero salgo más...y salgo fuera a despejarme...o voy a tomarme

345. algo...porque...ves mucha...yo creo que a las personas...que dejan también hijos...eso

346. debe ser un calvario...yo te lo diré...son...tu entrevistas a una persona que tiene los

347. hijos lejos y van a decir... "mira yo unos cuantos de euros y me voy a ver a mis

348. hijos...estoy yo ahorrando lo mínimo para traer mis hijos acá"...porque...hombre...

349. ./\#-aquí no se puede vivir así...con la mitad de tu alma allá...

Hay que recordar al lector, que el primer paso para hacer un análisis de membership categories es preguntarse cuál categoría de pertenencia el entrevistador ofrece al entrevistado en la pregunta que postula. Es una pregunta muy técnica, que sirve para identificar qué tipo de MC opera en la interacción entre entrevistador y entrevistado. ¿Qué tipo de MC puede operar en esta pregunta? ¿Qué tipo de expresión emocional hay?

Por ejemplo en la pregunta de la línea 333, el entrevistador ofrece una característica de esta $\mathrm{MC}$, que es la intimidad. Intimidad entendida cómo utilización emocional del espacio en que se produce esta performance, en este caso el locutorio, y cómo en este espacio afectan estas performance a los demás usuarios del mismo espacio. Un espacio que hay que compartir y que no siempre es "fácil". La misma entrevistada nos explica cómo en este espacio se vuelve más sensible (336), cómo su performance emocional está continuamente afectada por los demás, otras personas que comparten este espacio. Una continua performance de esta sensibilidad, que perjudica su ánimo, tanto que muchas veces sale del locutorio con un ánimo muy bajo, porqué se ve "tanta sensibilidad" (337). Y esto no se puede aguantar (337) y abandona este espacio. Esta característica de la intimidad, afecta a la membership category de esta relación con el Otro, en cuanto un individuo no puede aguantar tanta sensibilidad, y le afecta de manera totalmente emocional, tanto que los ánimos son muy bajos. Y esta sensibilidad no se puede definir anteriormente al análisis, porqué depende siempre de cada contexto en que la entrevistada se encuentra, muchas veces es "poca" y muchas veces es "demasiada", es una continua iteración que evoluciona. Y sólo a través del lenguaje es posible entenderla, sólo a través de este relato es posible encontrarla y comprenderla. La característica de la intimidad es fundamental para entender esta MC de la relación con el Otro. El lenguaje está siempre presente en esta performance, se introduce en la pregunta con la frase "teniendo una conversación" (335), y continua con "escuchas", "habla muy fuerte" (340), "cuando escucho" (343), "yo te lo diré" (346) y así siguiendo.

La comunicación es el elemento fundamental de nuestra performance emocional, sin ella sería imposible entenderla. Por ejemplo, la entrevistada nos describe cómo se articula una conversación con su mamá por el teléfono, u otras conversaciones a las que ella ha asistido en un locutorio (339-344). En esta comunicación, hablar y escuchar son las dos actividades principales, cargadas de emociones al máximo. Hay gente "que habla muy fuerte" (341), con la intención de poner el propio cuerpo en lo que se dice, y en un locutorio puedes escuchar estas performance emocionales, y estas conversaciones afectan tanto en el mundo online, como en el offline, en cuanto los usuarios del locutorio son testigos de estas conversaciones. "Sí...yo te mando todo...me quedo yo con lo mínimo..." (342) y esta performance tiene unas consecuencias no sólo online, sino también offline, la entrevistada sale de este espacio, se vuelve más sensible, y tiene que salir a "despejarse" (344), tanto que tiene que ir a tomarse 
algo, porqué estas performance le afectan demasiado su sensibilidad. Esta MC de relacionarse con el Otro y de compartir emociones, nos indica cómo en estos espacios sólo con el escuchar una determinada conversación provoca una serie de performance emocionales que hace que algunos usuarios dejen estos espacios. Son espacios cargados de acciones, de actividades (llorar, reír, gritar, etc.), de emociones. Este aspecto lo hemos entendido cuando entrevistábamos a estos usuarios y nos acercábamos a estos espacios. También estos espacios afectan emocionalmente a los investigadores, y por esta razón nos ha parecido oportuno poner en el titulo este aspecto muy frecuente, de llorar delante de una pantalla plana, justo para sintetizar lo que sucede en sitios tan emblemáticos como estos.

Antes hemos mencionado que gracias al concepto de performance de Judith Butler es posible meter el cuerpo en el lenguaje, sobre todo cuando se trata de comunicar emociones estando a miles de kilómetros de distancia, un ejemplo es lo de enviar un abrazo o un beso a una persona muy importante para nosotros, pero también transmitir algunas emociones muy difíciles de transmitir sólo a través de las palabras, y por esta razón muchas veces en un locutorio podemos escuchar a personas que hablan muy fuerte (341), que gritan o que utilizan palabras muy dulces (326). Con la intencionalidad de poder transmitir lo que le pasa corporalmente, poder decir algo más que unas simples palabras, introduciendo un elemento fundamental en la conversación que es el tono de la voz. No se puede separar cuerpo y mente, según el concepto de embodiment elaborado por Elizabeth Grosz (1995): el cuerpo existe y tiene que ir articulado a través del lenguaje, siempre junto con la mente. Y también es posible introducir este cuerpo en el lenguaje, expresando de otra manera lo que queremos decir. Este "nuevo" acto de habla, que es la performance, se realiza corporalmente y la simultaneidad de la producción y de la exteriorización de la expresión no sólo comunican lo que se dice, sino que muestra el cuerpo como el instrumento privilegiado de la expresión retórica. En el caso de una persona que tiene los hijos lejanos, es posible escuchar palabras como estas: "mira yo unos cuantos de euros y me voy a ver a mis hijos...estoy yo ahorrando lo mínimo para traer mis hijos acá" (347-348), por la razón que es imposible vivir físicamente en un sitio y tener la propia alma "allá" (349). Cuerpo y alma en sitios diferentes, y a través del lenguaje intentamos ordenarlas, ponerlos en su sitio, explicando dónde y en qué manera se encuentran. Intentar transmitir lo mejor posible en sus relatos, por esta cuestión la performance es tan importante cuando se trata de la relación entre emociones y lenguaje.

\subsection{Identidades emocionales en un mundo online/offline Extracto 3}

363. dinero?" "yo no, yo lo que gano me lo gano pa mi" entonces son casos muy diferentes... 364. -¿CÓMO AFECTA ESO A LA GENTE...CÓMO SE RELACIONA AHÍ...? POR 365. EJEMPLO...?

366. ./-afecta...bueno...a mi me da pena... ya te digo que salgo...y yo también pienso

367. que...todo eso...a ala inmigración...a los problemas económicos que cada país

368. tenemos...hace que muchos...muchos matrimonios se rompan...porque vienes aquí...y la

369. sol...la misma soledad...hace que te busques compañía...o sea hablar con gente...y te

370. olvides que allá hay gente que te...quiere y que...que has venido a por una meta...mucha 371. gente que...que se separa...la distancia mata...

372. - ¿Y LAS OTRAS PERSONAS CÓMO REACCIONAN? O SEA...TU SALES Y HAY 373. OTRA GENTE...¿NO?...

374. -las otras...pues mira...siguen llorando...y hablan de su país... "que si estuviera allá”...me 
375. iban a cuidar mejor" la verdad... y la música...es como un domingo...tu ven...porque en 376. el domingo hacen comida aquí de mi país...aquí de Perú...yo vengo un domingo ponen 377. música de mi país...y me transporto...como si estuviera un domingo en casa...o ahí en un 378. sitio...en un restaurante...de mi país...y me relajo y me lo paso...escucho música...y de ahí

379. que me apetezca tomarme una clarita...y comes algo de tu país...eso que la gente que se 380. queda ahí...pues mira...siguen sufriendo...

En esta MC de la relación con el Otro, hemos entendido cómo este cuerpo entra en el lenguaje también para explicar prácticas cotidianas cómo el envío de dinero a la propia familia. Una operación tan "terrena" cómo el envío de dinero se transforma en una performance emocional más, en cuanto se intenta darle un significado totalmente emotivo a este envío. De hecho, hay personas que "lo que gano lo gano pa mi" (363), y ejemplos que se quedan con lo mínimo para enviar lo más posible a la familia. Este uso totalmente emocional del envío del dinero, es una manera más de relacionarse con los demás, con los que se han quedado en el País de Origen, y es uno de los argumentos más tratados en las conversaciones diarias de los inmigrantes. Por el dinero se han venido a otro País, por el dinero sus familias no han podido acompañarlos, y siempre por el dinero, muchas veces pueden volver a vivir juntos o pueden fracasar en el intento. Así que eso del dinero es un argumento fundamental cuando se trata de estas performance emocionales. Muchas veces con la performance de enviar dinero a los que están en el País de Origen, el individuo se siente como si estuviera allá, y pudiera ocuparse de su propia gente. Es una práctica cotidiana que nos sirve para comprender esta performance emocional. En este caso es un elemento material que entra en el lenguaje, y que permite al entrevistado sentirse como "que si estuviera allá" (374).

En este extracto, el entrevistado introduce el último elemento que caracteriza esta membership category, la identidad emocional. Característica que nos recuerda mucho los trabajos de Ivan Leudar sobre los refugiados políticos (Leudar, 1998). De esta manera, hemos considerado la categoría de identidades emocionales en este extracto, en cuanto característica constituyente de la MC de la relación con el Otro. Es decir, que tal y como dice el MCA, no sólo tenemos categorías que nos permiten 'catalogar' a las personas de diferentes maneras, si no que estas categorías trabajan con implícitos y proveen de recursos no dichos que nos permiten interpretar 'más cosas' (Hutchby y Wooffitt, 2008), en nuestro caso las emocionales.

En la línea 346 emerge la identidad emocional de la madre que tiene los hijos lejos, y la entrevistada nos dice que si estuviera ella en esta situación, sería un calvario. Esta madre es inmigrante, tiene los hijos en su País de Origen y ella emigró por trabajo, así que también la define como trabajadora. Necesita enviar dinero a casa, enviar dinero a sus hijos. La entrevistada nos describe esta mujer según tres diferentes identidades, $\mathrm{y}$ lo hace siempre desde el punto de vista emotivo. Así que esta MC de la relación con el Otro, se identifica en ser madre, inmigrante y trabajadora. Estas tres diferentes identidades son entendidas en este relato como identidades emocionales. Son una performance emocional constante, en cuanto en el relato esta persona asume estas tres diferentes identidades según con cual aspecto emocional se enfrenta. La primera es una madre que extraña a sus hijos, y por esto llora, y por eso le parece que está viviendo un calvario (346). También es trabajadora, que espera tener unos cuantos euros para poder llevar sus hijos al mismo sitio en que ella vive (348). Por último inmigrante, que tiene el cuerpo en un sitio y el alma en otro. Estas tres identidades son 
complementarias y se relacionan entre si, pero cada una constituye una parte de esta performance emocional y alimenta la MC de esta relación con el Otro.

Por otro lado, la misma entrevistada asume otras identidades, es soltera e integrada. Se define soltera (352) en cuanto no tiene ninguna relación que le afecta emocionalmente, como por ejemplo la madre de antes que tiene hijos, y también se imagina que si hubiera tenido hijos, seguramente no hubiera aguantado esta lejanía, porqué hubiera sido una locura aguantar todo esto, como lo aguanta aquella madre, entonces aparece una continua performance entre tener una relación y no tenerla, y como ésta le influiría emocionalmente. También emerge la cuestión de la identidad, cómo ha asimilado (353) esta lejanía que no siempre es fácil de vivir. De hecho la entrevistada asume que "cada dos por tres" (353) entra en una fase de depresión y se pone a llorar, así que podemos comprender esta performance emocional en su totalidad, entre el haber asimilado y el no haber asimilado este factor de la lejanía y de la integración en la sociedad actual en que vive, esto le provoca depresión y llanto. Llama también la atención que la entrevistada utilice el termino "depresión" (354) para definir su particular identidad emocional. El término depresión es un término bastante nuevo en la arena discursiva, que hace cincuenta años no se hubiera utilizado con tanta frecuencia como ahora. Hipotéticamente la entrevistada hace cincuenta años hubiera utilizado diferentes emociones para definir el estado de animo en que se encontraba, cómo por ejemplo, los términos melancolía o nostalgia por su tierra en lugar de sentirse deprimida. Pero esto forma parte de la performance emocional, que tiene que ser entendida en el contexto particular en que aparece. Depresión es un termino que en la actual sociedad describe un particular estatus emotivo, una particular situación en un particular contexto, seguramente si la entrevistada hubiera utilizado términos como melancolía o nostalgia hubiera obtenido otros efectos que no los que ha provocado diciendo que le entra esta depresión.

Una identidad emocional que emerge muchas veces cuando se trata de analizar estas entrevistas a usuarios de locutorios, es la de inmigrante. El espacio del locutorio, es un espacio utilizado principalmente por inmigrantes, y con esta identidad se entrecruzan una serie interminables de emociones, cómo se ha podido entender en el análisis de la madre trabajadora inmigrante. Esta performance emocional se define e interacciona según muchos aspectos. Se trata de distinguir cuáles son las inferencias típicas de las categorías, como inmigrante y trabajadora, y las que en su conjunto vemos diferentes o especiales o específicas de la pantalla.

Primero, lo de las relaciones afectivas (368), los matrimonios se rompen en cuanto un inmigrante recién llegado se siente solo, la soledad es muy importante para un recién llegado, y esto hace si que si tu pareja se ha quedado en el País de Origen, tu busques compañía en el contexto actual en que te encuentras (369), olvidándote de quien te quiere allá, eso pasa porqué la "distancia mata" (371).

Hay matrimonios que duran y matrimonios que se rompen (368), hay quien sigue añorando su País por toda la vida pero también quien se olvida rápido. Son unas continuas performance emocionales que tienen que ver con la construcción y deconstrucción de estas identidades emocionales que nacen de la relación entre individuo y el contexto en que habita.

Esto de la distancia que mata es un continuo conflicto para un inmigrante, en cuanto su vida se divide entre mundo online y mundo offline. El mundo online es su mundo originario, allí está lo del País de Origen, estas actividades cotidianas que le permiten comunicar con su gente que sigue viviendo allí.

En nuestro caso, este conflicto lo entendemos cómo conflicto online/offline, en cuanto en estos específicos espacios, los locutorios, se generan dinámicas totalmente novedosas. Los 
usuarios de estos espacios comunican con su País de Origen, y esto les permite borrar las distancias de manera online, pero también en estos espacios se encuentra gente que ha tenido las mismas experiencias, que ha tenido que abandonar su hogar, dejar el mismo País, y por esta razón, también de manera offline, parecen sentirse emocionalmente en casa. Se escucha música del país de origen (377), se come algún producto de allí (379), y la entrevistada se transporta (377), esta es una performance emocional en su totalidad. Ha pasado de llorar por no estar en su patria, a estar contenta porqué se siente como en casa cuando utiliza el locutorio. Son conflictos que alimentan esta performance emocional entre el mundo on-line/off-line.

\section{CONCLUSIONES}

En el contexto de los locutorios, hay prácticas que son una elección continua y cotidiana, que tienen una función importante y esencial para la vida social y la relación con los demás. La importancia de estos lugares es fundamental para la relación entre los individuos, y las performance allí están a la orden del día, no tanto por el uso de las TIC que se hace, sino por el contacto humano, las relaciones con el Otro. En una época de alienación individual, comprender que aún es importante sentirse miembro de un grupo, de una comunidad, y que esto es posible con la simple acción de ir al locutorio, y transportarse "allá", es esencial para comprender la importancia emocional que conlleva un sitio como este. En estos espacios las relaciones sociales pueden ser invertidas para crear el espacio heterotópico (Foucault, 1984:422). Porque también a nivel virtual estos lugares se constituyen en espacios emocionales donde dos personas lejanas se encuentran, en algún chat o en un cable de teléfono, pero parece que de aquel encuentro, exista un lugar físico, una formación de lugar, que traslada a ambas personas la potencia de esta performance. Este entorno virtual, totalmente construido por el discurso, es una continua performance emocional.

El locutorio, justo por aquellas características que hemos descrito anteriormente, genera una serie de emociones en el usuario de estas TIC que difícilmente podemos encontrar en otro sitio. Los individuos están emocionalmente implicados, en cuanto hay una intimidad con el interlocutor que está al otro lado del hilo telefónico o de la pantalla, y que le permiten aislarse de aquel contexto. Esto no quiere decir que se olviden de que están en un contexto publico, es decir que su intimidad puede estar expuesta o en peligro. La performance emocional permite estar en otro sitio, hablando, intimando con otra persona, pero siempre alerta del contexto público.

Como comentamos en anteriores trabajos (Gil-Juárez, 1999; Belli, Íñiguez, 2008; Belli, S, Harré, R, Íñiguez, L. 2009), las emociones tienen una fuerte relación con el lenguaje. Emocionarse significa poner algo en común con los demás. Gracias al concepto de performance, elaborado por Judith Butler, hemos comprobado que las emociones no se prestan fácilmente al proceso de categorización, sino que están en constante evolución, cumplen continuamente un proceso de iteración, y lo hacen a través del lenguaje, natural y subjetivo.

Hemos entendido en este artículo el concepto de performance emocional como algo que nos sirve para argumentar la tesis de que las emociones van cambiando en el discurso. Seguramente hay una infinita variedad de emociones que se producen cuando las personas utilizan las TIC para comunicarse con los demás. Y estas emociones pueden ir desde el 
aburrimiento hasta la felicidad, del enfado hasta el amor, pero esto sólo podemos saberlo a través del lenguaje. Este lenguaje produce lo que denominamos una performance emocional.

Igualmente hemos intentado introducir una diferente manera de entender las emociones, a través el concepto de performance, y una nueva manera de acercarnos al trabajo empírico, gracias al Membership Categoriszation Analysis según la perspectiva de Manchester. De esta manera, hemos ofrecido una propuesta diferente para investigar el tema de las emociones en las TIC. Introduciendo una nueva manera de "hacer" análisis a través de una herramienta metodológica que nos permita ver en el discurso, cómo las emociones evolucionan e interaccionan, a través de las actividades, de las acciones, acorde con el cambio en el estudio de las emociones en Ciencias Sociales de los últimos años.

Una nueva manera de acercarnos a los datos empíricos, en cuanto nosotros mismos estamos en una sociedad cambiante y cambiada a través el uso de las TIC.

Esta investigación ha sido realizada gracias al apoyo del Ministerio de Educación y Ciencia a través del proyecto financiado: "Impacto psicosocial y cultural de las innovaciones tecnocientíficas: transformaciones y cambios promovidos por las Tecnologías de la Información y la Comunicación (TIC)" (SEJ2006-15655-C02-01).

\section{BIBLIOGRAFÍA}

BARICCO, A. (2008): Los bárbaros: Ensayo sobre la mutación, Barcelona, Anagrama. BAUDRILLARD, J. (1990): Seduction, New York, St. Martin's.

BELLI, S. y GIL, A. (2009): Llorar delante de una pantalla plana. Emociones compartidas en un locutorio. XI Congreso Nacional de Psicología Social, Tarragona, 01-03 de octubre de 2009.

BELLI, S.; HARRÉ, R. e ÍÑIGUEZ, L. (2009): "Emociones en la tecnociencia: la performance de la velocidad", Prisma Social, 3, pp. 1-41.

BELLI, S. e ÍNIIGUEZ, I. (2008): Emociones y lenguaje: el concepto de "performance" en el Membership Categorization Analysis, VIII Congreso de Lingüística, Madrid, 25-28 de junio de 2008.

BELLI, S. e ÍÑIGUEZ-RUEDA, L. (2008): "El estudio psicosocial de las emociones: Una revisión y discusión de la investigación actual", PSICO, 39(2), pp. 139-151.

BELLI, S., et al. (2007): La producción de conocimiento en torno a los lugares de acceso público a las TIC: una aproximación a los locutorios desde las ciencias sociales, X Congreso Nacional de Psicología Social, Cádiz, 26-28 de septiembre de 2007.

BUTLER, J. (1993): Bodies that matter: On the discursive limits of sex, London, Routledge.

- (1997): Excitable speech: A politics of the performative, London, Routledge.

- (2003): Gender trouble. Continental Feminism Reader, London, Routledge.

FELIU, J. (2006): “Adicción o violencia: dilemas sociales alrededor de las nuevas tecnologías y los jóvenes”, en GIL, A. y VALL-LLOVERA, M. (eds.) Jóvenes en cibercafés: la dimensión física del futuro virtual, Barcelona, Editorial UOC.

FOUCAULT, M. (1984): El uso del placer. Historia de la Sexualidad II. Madrid, Siglo XXI.

GARAY, A.; MARTÍNEZ, L. M. y PEÑARANDA, M.C. (2004): "Habitando en los locutorios: prácticas cotidianas e inmigración". Cuarto congreso sobre la inmigración en España. Ciudadanía y Participación, Girona, 10-13 de noviembre de 2004.

GIL-JUÀREZ, A. (1999): Aproximación a una teoría de la afectividad, Tesis doctoral, Barcelona, Universitat Autonoma de Barcelona. http://www.tdx.cat/TDX-0331108-110854

- (2006): "Consumir TIC y producir Tecnologías de Relación. Aproximación teórica al papel del consumo de TIC en jóvenes", en GIL, A. y. VALL-LLOVERA, M. (eds.) Jóvenes en cibercafés: la dimensión física del futuro virtual, Barcelona, Editorial UOC. 
- (2009): Consumption as an Emotional Social Control Device. Theory and Psychology. 19(6), pp. 837857.

GIL-JUÀREZ, A. y FELIU, J. (2004): "Consum i producció de noves tecnologies de relació per part del joves catalans", en REGUILLO, R.; FEIXA, C.; VALDEZ, M.; GÓMEZ-GRANELL, C. y PÉREZISLAS, J. (ed.) Temps d'hibrids. Entresegles, Joves, Catalunya - Mèxic., Ciudad de México, Instituto Mexicano de la Juventud, Secretaría General de Juventud, pp. 71-91.

- (en prensa). "Consecuencias de las nuevas formas de trabajo: jóvenes, migraciones y TIC", en ÁlVARO, J. y AGUllo, E. (eds.), Psicología Social y Organizaciones, Guadalajara, México, Universidad de Oviedo y Universidad de Guadalajara.

GREGSON, N., y ROSE, G. (2000): "Taking Butler elsewhere: Performativities, spatialities and subjectivities", Environment and Planning D, 18(4), pp. 433-452.

GROSZ, E. (1995): Space, Time and Perversion: Essays on the Politics of Bodie, New York, Routledge. HARAWAY, D. (1990): A manifesto for cyborgs: Science, Technology, and Socialist, New York, Routledge.

HUTCHBY, I. y WOOFFITT, R. (2008): Conversation Analysis (Second Edition), London, Polity Press. ILLOUZ, E. (2007): Cold intimacies: The making of emotional capitalism, London, Polity.

LEUDAR, I. (1995): "Reporting political arguments", en Van Eemeren (ed.), Reconstruction and Application: Proceedings of the Third Conference on Argumentation, Amsterdam, Sic Sat, pp. 42-59.

- (1998): "Who is Martin McGuiness 1: On contextualizing reported political talk". Dialogue Analysis, 6, pp. 217-224.

LEUDAR, I., y NEKVAPIL, J. (1998): "On the emergence of political identity in the czech mass media: The case of the democratic party of sudetenland". Czech Sociological Review, 6(1), pp. 43-58.

MARTÍNEZ, L.M. y PEÑARANDA, M.C. (2005): "Inmigración y construcción de espacios transnacionales a través del uso de las TIC", en SABUCEDO, J.M., ROMAY, J. y LÓPEZ-CORTÓN, A. (eds.). Psicología Social y problemas sociales. Psicología Política, Cultura, Inmigración y Comunicación Social, Madrid, Biblioteca Nueva, pp. 177-183.

SACKS, H. (1992): Lectures on conversation: Vol 1 \& 2. Oxford, Basil Blackwell.

WATSON, D. R. (1987): "Interdisciplinary considerations in the analysis of pro-terms". Talk and Social Organisation, 5, pp. 261-289.

\section{Breve currículo:}

\section{Simone Belli}

Investigador postdoctoral en la Universidad Autónoma de Madrid. Doctor en Psicología Social por la Universidad Autónoma de Barcelona, mención Doctor Europeo con la tesis 'Emociones y lenguaje' dirigida por Lupicinio Íñiguez Rueda. Fue becario FPU del Ministerio de Educación y Ciencia. Actualmente es miembro del Grupo consolidado de investigación MIRCo (Multilingüismo, Identidades Sociales, Relaciones Interculturales y Comunicación), del Grupo de Estudios Sociales en Ciencia y Tecnología (GESCIT), y del Centro de Estudios del Discurso (CED).

Adriana Gil JuárezProfesora Lectora de Psicologia Social en la Universitat Rovira i Virgili. Licenciada en Psicología por la Universidad Nacional Autónoma de México. Magíster y Doctora en Psicología Social por la Universitat Autònoma de Barcelona. Coordina el grupo de investigación JovenTIC, que tiene como principal objetivo el estudio de las prácticas en torno al uso, apropiación y consumo, que los jóvenes hacen, de las TIC. Así, se interesa por el conjunto de significados que los jóvenes construyen colectivamente en estas prácticas y su relación con la construcción de su identidad, especialmente las identidades generizadas. 